\title{
Efficient Design of a High Speed PMSM Drive for Electric Vehicle Application Using Real Time Simulator
}

\author{
Amin Zabihinejad ${ }^{1}$, Philippe Viarouge ${ }^{2}$, Simon Roy ${ }^{3}$, and Jéröme Cros ${ }^{4}$ \\ ${ }^{1}$ Department of Electrical Engineering, Laval University, amin.zabihinejad.1@ulaval.ca \\ ${ }^{2}$ Department of Electrical Engineering, Laval University, philippe.viarouge@gel.ulaval.ca \\ ${ }^{3}$ Department of Electrical Engineering, Laval University, simon.roy.16@ulaval.ca \\ ${ }^{4}$ Department of Electrical Engineering, Laval University, jerome.cros@gel.ulaval.ca
}

\begin{abstract}
In this paper, a novel approach has been employed in order to investigate the efficient design of a vector control drive of a high speed PMSM for automotive application using analytical method and real time simulator. Most of vector control drives set the $d$-axis current to zero to decrease the magnitude of the source current and working with unit power factor. However, the optimal d-axis current is dependent to the motor specifications. An optimal estimation scheme has been proposed and tested in order to work with maximum efficiency based on the operation point. In the high speed application, because of high losses density and low capacity of heat dissipation, the importance of working in maximum efficiency point will be clear more and more. Also, measurement of inverter losses is very complicate in the analytical approach. In this paper, the precise model of the switches has been implemented using real time simulator. Real time simulator, is a complex electronic circuit which is composed of CPUS, FPGA, etc.-is known as an efficient tool obtains very exact model of the IGBTs in order to have the precise losses model of the motor and drive. Both of the analytical approach and real time simulator prove the validity of the optimal estimation scheme.
\end{abstract}

\section{Keywords}

vector control drive, PMSM, loss minimization, real time simulator, electric vehicle

\section{INTRODUCTION}

Demands to use Permanent Magnet (PM) motors are increasing more and more because of its high performance and ideal control characteristics [Ta and Hori, 2001; Blaabjerg and Pedersen, 1993]. The recent development of rare-earth permanent magnet (PM) materials, power electronic components and simulators have contributed to new energy efficient, high performance electric drives that use modern PM synchronous motors [Ta and Hori, 2001; Blaabjerg and Pedersen, 1993; Joorabian et al., 2009]. Regarding to the appearance of rare-earth PMs, PM motors obtain higher efficiency, power factor, power density and better dynamic performance than other types of conventional motors without sacrificing reliability [Blaabjerg and Pedersen, 1993]. The most important advantage of PM motors is their ability to work in the wide constant power speed range [Joorabian et al., 2009] which causes a vast amount of attention to the PM motors in a different types of automotive applications and electric vehicles. For industrial applications such as electric vehicles, high efficiency is very important to increase the range per battery charge under all operat- ing conditions [Monga et al., 2012].

In compact electric vehicles, PMSMs are usually designed with maximum torque per volume. Therefore, their power density is high. This characteristic leads to high losses density in the machine. The heat dissipation is a complex issue in this type of motor. Decreasing the losses and working at optimal point to generate minimum losses will be an effective control strategy for this type of application.

In this paper, a novel loss minimization aspect has been introduced and evaluated in order to find the optimal losses point of vector control drive and PM motor for all operating points. The controller tries to minimize the overall losses from the motor and inverter. The researchers proposed different minimumloss control strategies previously. All of these methods are divided in three groups; Search method [Ta and Hori, 2001], Loss model method [Blaabjerg and Pedersen, 1993] and Power factor method [Joorabian et al., 2009].

The losses model approach has been employed in order to estimate the losses energy in the different operating conditions. The precise losses model of the PM motor and VSI has been derived using analytical analysis.

The validity of the controller performance has been evaluated using analytical approach and real time 
simulator. Analytical approach estimates the precise value of the losses, but it is very difficult to implement in the simulation and usually it passes up. Real time simulators have resolved this problem.

In this research, a real time simulator has been employed to verify the simulation results. Real time simulators are composed of software and hardware sections. OPAL-RT Company introduces the RT-Lab as the software which compiles the model in order to implement in the hardware section. It is fully integrated with Matlab/Simulink and compiles the generated model in the sympower library. OP4500 is a real time hardware simulator which is used to execute the power electronic models. OP4500 utilizes the exact IGBT and Diode and power system elements model using FPGA technology. Moreover, other parts of the simulation are executed by the powerful CPUs of OP4500. The Real time simulator helps us to execute the very complex power electronic model in real time to evaluate the dynamic setting of the controller and power quality of the converter [Monga et al., 2012]. This is a valuable step in order to design the high power converters which are very expensive and time consuming to implement.

\section{THE HARMONIC LOSSES MODEL IN PMSM MOTORS}

One of the advantages of PMSMs is their higher efficiency than induction motors when used in adjustable speed drives [Monga et al., 2012]. G. Slemon (1990) proposed an analytical approach in order to calculate the fundamental losses of PMSM [Slemon and Liu, 1990]. In 2008 a theoretical study has been done to calculate the effect of PWM parameters on the iron losses [Ruifang, 2008]. Regarding to the copper losses, measuring the effective value of the current is acceptable.

$$
P_{C u}=3 R_{s} \sum_{n=1}^{\infty} I_{n}^{2}
$$

The core losses in the machine consist of two components, i.e., hysteresis and eddy current losses. Both types of core losses are due to time variation of the flux density in the core. The value of flux density is not equal in the yoke and tooth section.

$$
\left\{\begin{array}{l}
P_{h t}=\frac{1}{\gamma_{F e}} K_{h t} \sum_{n=1}^{\infty} n \omega_{s} \lambda_{t, n}^{\beta} \\
P_{h y}=\frac{1}{\gamma_{F e}} K_{h y} \sum_{n=1}^{\infty} n \omega_{s} \lambda_{y, n}^{\beta}
\end{array}\right.
$$

We consider that $\lambda_{y, n}=m \lambda_{t, n}$ where $m$ is dependent to the mass proportion of tooth to yoke. Therefore,

$$
P_{h}=K_{h} \sum_{n=1}^{\infty} n \omega_{s} \lambda_{t, n}^{\beta}
$$

Similarly, for the eddy current losses, we will have the equation below:

$$
P_{e}=K_{e} \sum_{n=1}^{\infty}\left(n \omega_{n}\right)^{2} \lambda_{t, n}^{2}
$$

\section{THE LOSSES IN THE VOLTAGE SOURCE INVERTER}

The inverter losses are often separated in conduction losses and switching losses [Casanellas, 1994]. The conduction losses $\left(P_{c o n}\right)$ are the losses of the IGBT or diode in the ON position.

The switching losses are the most important proportion of the inverter losses. They are directly related to the switching frequency. Also, in order to calculate the precise value of the switching losses, it is necessary to consider the ON/OFF fitting curve of the switch. Most of the previous researches employed the approximation method to calculate the switching losses. In this research, the exact model of the IGBT has been used by the real time simulator.

The total inverter losses will be written as below [Casanellas, 1994; Zabihinejad and Moghani, 2008],

$$
\begin{aligned}
& \left.P_{i n v}=6 f_{s} \sum_{n=1}^{f_{c}}\left\{K_{I G B T} i\left(\frac{n}{f_{c}}\right)^{\beta_{I G B T}}+K_{\text {diode }} i\left(\frac{n}{f_{c}}\right)^{\beta_{\text {diode }}}\right)\right\} \\
& +6 f_{s}\left\{\int_{0}^{T} V_{I G B T, \text { on }}(t) i(t) d t+\int_{0}^{T} V_{\text {diode, on }}(t) i(t) d t\right\}
\end{aligned}
$$

\section{DYNAMIC MATHEMATICAL MODEL OF PMSM LOSSES}

The equivalent circuit of q-axis and d-axis of the PMSM are shown in Figure 1 respectively. These are the most precise circuit model which are conventionally used to calculate the copper and iron losses in the same platform. It is clear that the iron losses and mechanical behavior of PMSM are not independent parameters. The armature current which is passed through $R_{s}$, is converted to electromagnetic torque ( $I_{q m}$, $\left.I_{d m}\right)$ and iron losses $\left(I_{q F e}, I_{d F e}\right)$.

In the steady-state condition, the currents of $I_{d F e}, I_{q F e}$ will be as below:

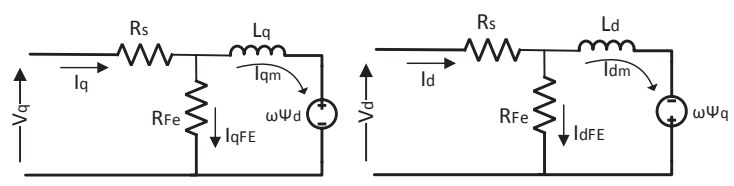

Fig. 1 q/d-axis equivalent circuit of PMSM 
$\left\{\begin{array}{l}I_{d F e}=\frac{-\omega L_{q} I_{q m}}{R_{F e}} \\ I_{q F e}=\frac{\omega L_{d} I_{d m}+\omega \lambda_{m}}{R_{F e}}\end{array}\right.$

where $I_{d F e}=I_{d}-I_{d m}, I_{q F e}=I_{q}-I_{q m}$.

Using these definitions, the power equations are:

$P_{C u}=R_{s}\left(I_{d}^{2}+I_{q}^{2}\right)$

$P_{F e}=\frac{\omega^{2} L_{q}^{2} I_{q m}^{2}}{R_{F e}}+\frac{\left(\omega L_{d} I_{d m}+\omega \lambda_{m}\right)^{2}}{R_{F e}}$

$P_{\text {motor }}=P_{\text {out }}+P_{C u}+P_{F e}$

Therefore, the input power versus the current is written as below:

$P_{i n}=K_{m} \Omega^{3}+3 R_{s}\left(I_{q}^{2}+I_{d}^{2}\right)+K_{h} \omega \lambda^{2}+K_{e} \omega^{2} \lambda^{2}$

where,

$\lambda^{2}=L_{d} I_{d m}^{2}+L_{q} I_{q m}^{2}+L_{d} I_{d m} \lambda_{f}+\lambda_{f}^{2}$

$I_{q m}=\frac{R_{F e}^{2} I_{q}-\omega L_{d} R_{F e} I_{d}+\omega \lambda_{f} R_{F e}}{R_{F e}^{2}-\omega^{2} L_{d} L_{q}}$

$I_{d m}=I_{d}-\frac{\omega L_{q}\left(R_{F e} I_{q}-\omega L_{d} I_{d}+\omega \lambda_{f}\right)}{R_{F e}^{2}-\omega^{2} L_{d} L_{q}}$

\section{FINDING THE OPTIMAL OPERATING POINT}

In PMSM motors, the electromagnetic torque is dependent to $I_{q m}$. Therefore, in each operating point of the motor, there is an optimum value for $I_{d m}$ which generates the minimum motor losses. In order to find the optimum point, we need to have the total value of power from the inverter input. The total input power will be:

$P_{\text {Total }}=P_{\text {motor }}+P_{\text {inverter }}$

The minimum value of the total power is determined by the equation below.

$\frac{d P_{\text {Total }}}{d I_{d}}=0$

The optimum value of $I_{d}$ will be determined solving the above equation. It a nonlinear equation which needs a numerical approach to solve.

\section{SIMULATION AND RESULTS ANALYSIS}

In this research, a 6-IGBT module, from Fuji Electric

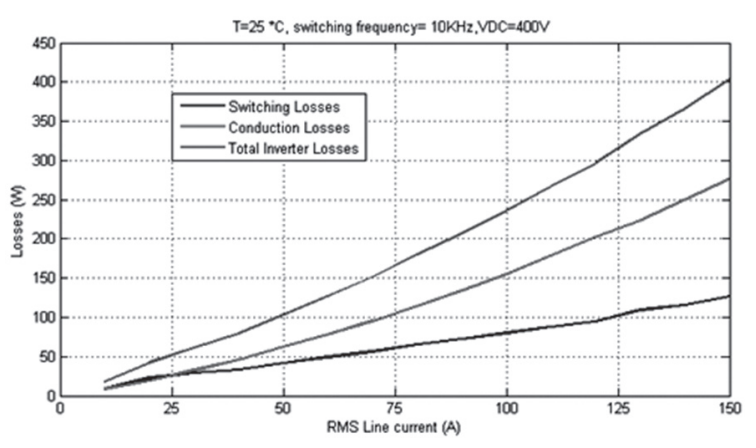

Fig. 2 The Inverter Losses versus line current

Company, has been used. The switching losses and conduction losses of the inverter versus the phase current is shown in Figure 2. The parameters of the semiconductor switches in the IGBT pack have been extracted from the data sheets of Fuji Electric module. These values are true when the switching frequency is $10 \mathrm{kHz}$, DC link voltage is $400 \mathrm{~V}$ and the ambient temperature is $25^{\circ} \mathrm{C}$. Increasing the line current leads to increase the inverter losses.

The parameters of a PMSM that has been prepared for a Formula Student Electric Vehicle Competition are listed in Table 1. The field oriented control algorithm including the loss minimization algorithm is implemented in MATLAB/SIMULINK and Real-Time Simulator. The FOC algorithm with loss minimization algorithm is shown in Figure 3. The electromagnetic

Table 1 PMSM Parameter

\begin{tabular}{ccc}
\hline PMSM Parameter & Value & Units \\
\hline Number of pole pairs & 4 & \\
\hline Stator resistance & 0.0533 & $\Omega$ \\
\hline Core loss resistance & 150 & $\Omega$ \\
\hline Rated speed & 12000 & $\mathrm{RPM}$ \\
\hline Rated torque & 8 & $\mathrm{Nm}$ \\
\hline Rotor flux linkages & 0.0239 & $\mathrm{~Wb}$ \\
\hline Rated peak voltage per phase & 400 & $\mathrm{~V}$ \\
\hline $\mathrm{Ld}$ & 0.00017 & $\mathrm{H}$ \\
\hline $\mathrm{Lq}$ & 0.00017 & $\mathrm{H}$ \\
\hline
\end{tabular}

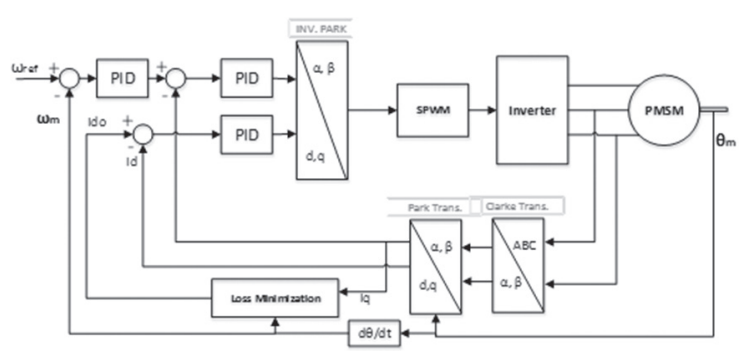

Fig. 3 FOC strategy for PMSM with loss minimization algorithm 
design of the PMSM has been done using Flux-3D software. It is possible to calculate the iron losses versus mechanical speed using electromagnetic software. The inverter losses are directly calculated using the SIMULINK system.

The losses of the PMSM and drive versus d-axis current in the nominal condition are shown in Figure 4. It is clear that optimal control is not happening in the zero value of $I_{d}$. The value of optimal $I_{d}$ is calculated based on the value of speed, q-axis current and motor parameters. Figure 5 shows the optimal Motor losses for different value of the speed and $I_{d}$. The optimal $I_{d}$ has been calculated by solving the nonlinear equations of the loss minimization algorithm which was explained in the previous section.

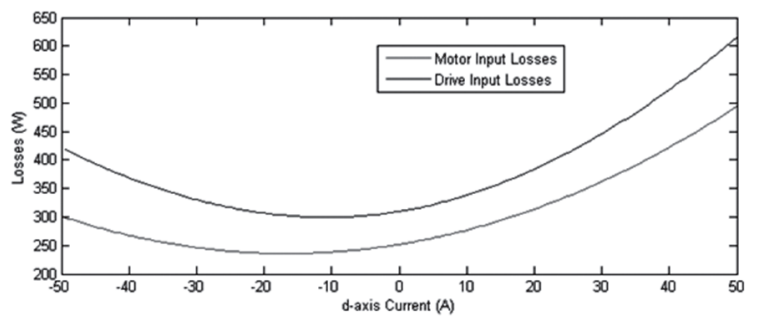

Fig. 4 The PMSM and drive losses versus $I_{d}$

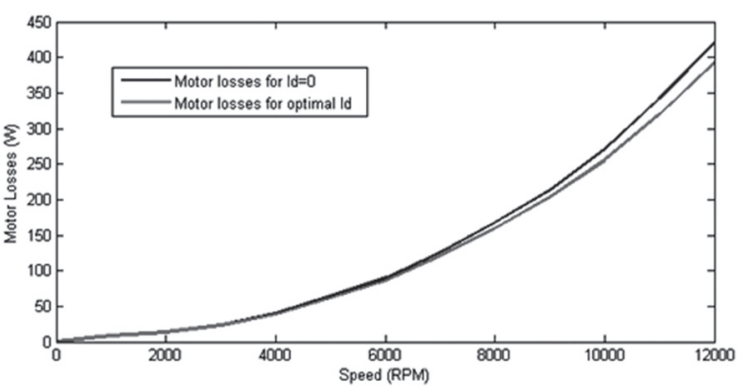

Fig. 5 PMSM power losses with loss minimization algorithm

\section{STEP RESPONSE OF FOC WITH LOSS MIN- IMIZATION ALGORITHM}

The step response of the FOC algorithm with loss minimization strategy has been investigated in Simulink/MATLAB. The step response is a suitable criterion to evaluate the performance of algorithm.

Figure 6 shows the step response of the speed and electromagnetic torque. Control system keeps the electromagnetic torque at maximum value in transient state. The variations of q-axis and optimal d-axis current in the step response with loss minimization algorithm are shown in Figure 7. The proposed loss minimization algorithm chooses the optimal value of d-axis current in transient and steady state condition. The results prove that increasing the value of electromagnetic torque increases the optimal value of d-axis
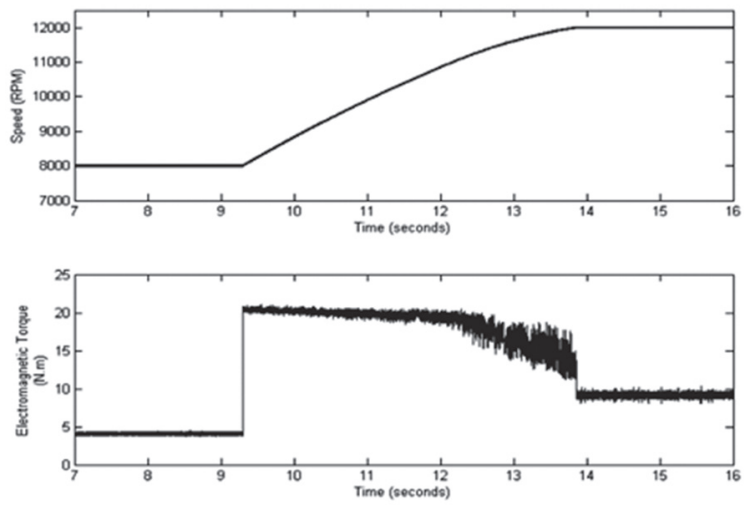

Fig. 6 Step response of the speed and torque
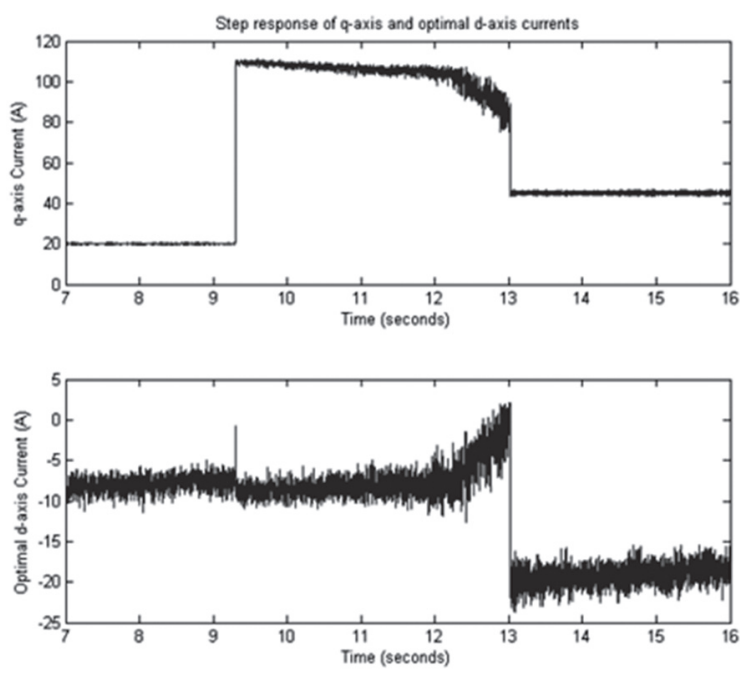

Fig. 7 Step response of $\mathrm{q}$ and d-axis currents with loss minimization algorithm

current.

\section{SIMULATION WITH REAL TIME SIMULA- TOR}

Real-time simulator (RTS) is an effective tool of virtual prototyping used to study the dynamics of a physical system prior to start the actual hardware development [Zheng et al., 2009; Le-Huy et al., 2006]. Recently, RTS has been utilized by engineers in various industries such as aviation [Zheng et al., 2009], power systems [Le-Huy et al., 2006], networking [Xiaobo et al., 2009], automotive [Tavernini et al., 2009], traffic management [Maroto et al., 2006], and medicine [Lerotic et al., 2009].

The implementation of power electronic converters is very expensive and time consuming, especially in high power applications. In electric vehicle applications, the stability and reliability of the controller performance in the various operating conditions is an important point of the design process. Real time simulators are known as a very effective approach to verify 
the model dynamics and controller settings validation. In this research, OP4500, a real time simulator from OPAL-RT Company, has employed to evaluate the controller design. The RTSs often have two main sections; software and hardware. The software is a computer program which connects the simulation files to the hardware. OPAL-RT introduces the RT-Lab as a software that receives the Matlab/Simulink files and translates them in order to load and execute. The OPAL-RT hardware is composed of two main sections; CPU and FPGA. The power system and power electronic components are directly implemented in FPGA section. The RT-LAB uses the precise model of IGBT, diodes and other power electronic components. The control system component is executed by the CPU section. All simulation sectors must execute in real time. Figure 11 shows the OP4500 and the setup which was prepared for this research.

In order to implement the FOC drive in the real time simulator, the components are divided in to two sections. Power electronic components are implemented in FPGA section in order to generate fast response for real time performance. PMSM machine and controller are implemented in the CPU section.

\section{REAL TIME SIMULATION RESULTS}

Figure 8 shows the OP4500, the real time simulator which is used in this project. The OP4500 has two independent FPGA modules supporting 24 power electronic switches each. The FPGA modules execute the exact model of the IGBTs and diodes in real time, causing a real time simulation with precise results.

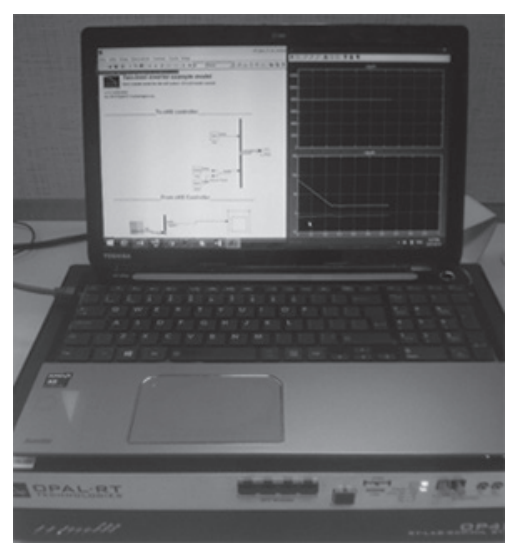

Fig. 8 Implementation of the drive with OP4500

Figure 9 shows the step response of the mechanical speed and electromagnetic torque. The waveform of the voltage in RTS and Simulink are the same except for the high frequency spikes. RTS uses the more precise model for IGBTs. The oscillations which are seen in the voltage waveform are visible when the exact
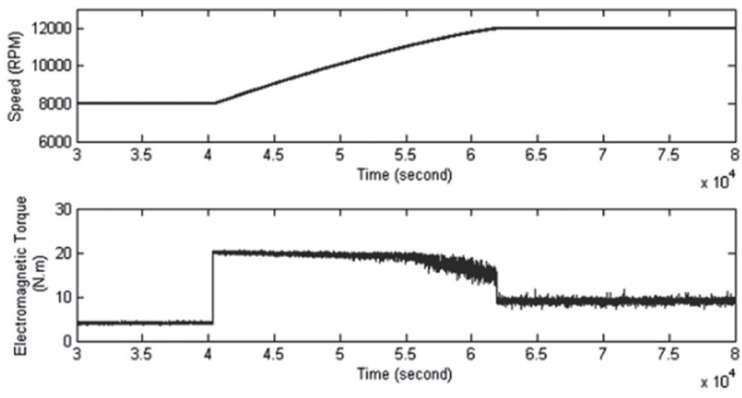

Fig. 9 Step response of speed and Torque in RTS
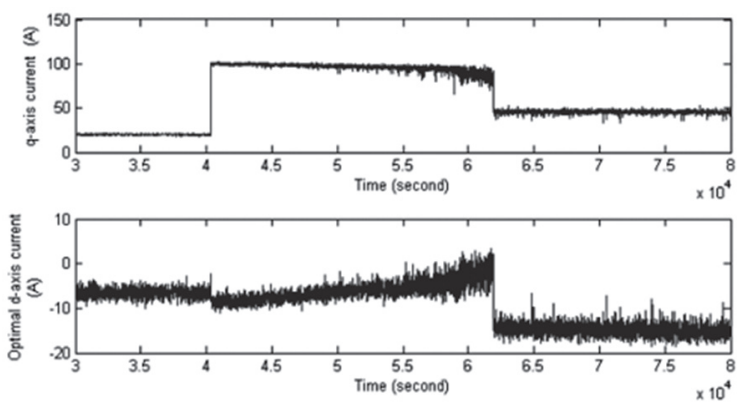

Fig. 10 Step response of $q$ and optimal $d$ axis currents in RTS

IGBT model is used. The step response of q-axis current and optimal d-axis current are shown in Figure 10.

\section{CONCLUSION}

In this paper, an effective design approach has been investigated in order to design an efficient vector control drive of a high speed PMSM for electric vehicle application. Losses minimization strategy for a high speed PMSM has been simulated and evaluated using two different approaches. The concept of vector control drive with losses minimization algorithm is verified by the Matlab/Simulink and real time simulator results. Real time simulator is an effective and valuable approach to demonstrate the validation of the controller setting in power electronic design, especially in high power applications [Tavernini et al., 2009; Maroto et al., 2006; Lerotic et al., 2009]. The results of the controller have been compared with conventional drive which sets the d-axis current to zero. The simulation results proved that the losses minimization algorithm decreases the motor losses and increases the overall efficiency of the motor and drive. Implementation of high speed PMSM, especially in electric vehicle technology is very expensive and risky. Real time simulator helps us to evaluate the simulation results. The OP4500 - OPAL-RT simulator was used in this research. The results with RTS proved the simulation results. 


\section{Acknowledgment}

The authors would like to acknowledge the active participation and financial support of the OPAL-RT Company and the department of electrical engineering of Laval University.

\section{References}

Blaabjerg, F., and J. K. Pedersen, An integrated high power factor three-phase AC-DC-AC converter for AC-machines implemented in one microcontroller, IEEE Power Electronics Specialists Conference, 285-292, 1993.

Casanellas, F., Losses in pwm inverters using igbts, IEE Proceedings-Electric Power Applications, Vol. 141, No. 5, 235-239, 1994.

Joorabian, M., A. Zabihinejad, and K. Malekian, Practical aspects of constructing a wind Ironless axial flux direct-drive generator, International Review of Electrical Engineering, Vol. 4, No. 2, 235-241, 2009.

Le-Huy, P., S. Guerette, L. Dessaint, and H. Le-Huy, Dual-step real-time simulation of power electronic converters using an FPGA, Proceedings of IEEE International Symposium on Industrial Electronics, 1548-1553, 2006.

Lerotic, M, S. L. Lee, J. Keegan, and G.-Z. Yang, Image constrained finite element modelling for realtime surgical simulation and guidance, Proceedings of Biomedical Imaging: From Nano to Macro, 1063-1066, 2009.

Maroto, J., E., Delso, J. Felez, and J. Cabanellas, Real-time traffic simulation with a microscopic model, IEEE Transactions on Intelligent Transportation Systems, Vol. 7, No. 4, 513-527, 2006.

Monga, M., M. Karkee1, S. Sun, L. K. Tondehal, B. Steward, A. Kelkar, and J. Zambreno, Real-time simulation of dynamic vehicle models using a highperformance reconfigurable platform, Procedia Computer Science, 1-10, 2012.

Ruifang, L., C. C. Mi, and D. W. Gao, Modeling of eddy-current loss of electrical machines and transformers operated by pulsewidth-modulated inverters, IEEE Transactions on Magnetics, Vol. 44, No. 8, 2021-2028, 2008.

Slemon, G.R., and X. Liu, Core losses in permanent magnet motors, IEEE Transactions on Magnetics, Vol. 26, No. 5, 1653-1655, 1990.

Ta, C. M., and Y. Hori, Convergence improvement of efficiency-optimization control of induction motor drives, IEEE Transactions on Industry Applications, Vol. 37, 1746-1753, 2001.

Tavernini, M., B. Niemoeller, and P. Krein, Real-time low-level simulation of hybrid vehicle systems for hardware-in-the-loop applications, Proceedings of
Vehicle Power and Propulsion Conference, 890895, 2009.

Xiaobo, X., Z. Kangfeng, Y. Yixian, and X. Guoai, A model for real-time simulation of large-scale networks based on network processor, Proceedings of the Broadband Network Multimedia Technology, 237-241, 2009.

Zabihinejad, A., and J. S. Moghani, Design \& implementation of a novel vector-controlled drive by direct injection of random signal, Vehicle Power and Propulsion Conference, 2008.

Zheng, S., J. He, and J. Han, An optimized distributed real-time simulation framework for high fidelity flight simulator research, Proceedings of International Conference on Information and Automation, 1597-1601, 2009.

(Received July 9, 2015; accepted October 14, 2014) 